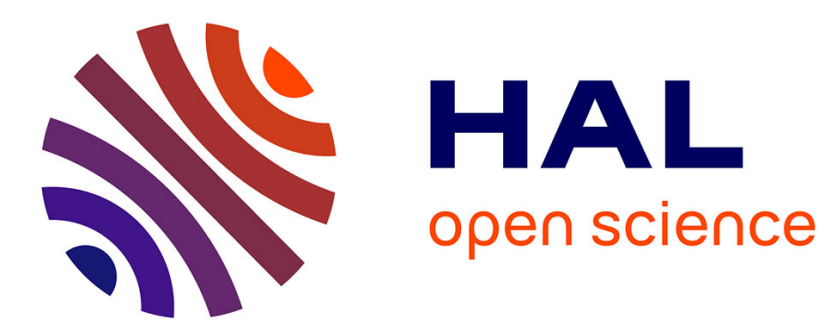

\title{
Finite element solutions of axisymmetric Euler equations for an incompressible and inviscid fluid
}

\author{
J.H. Saiac
}

\section{To cite this version:}

J.H. Saiac. Finite element solutions of axisymmetric Euler equations for an incompressible and inviscid fluid. RR-0999, INRIA. 1989. inria-00075560

\section{HAL Id: inria-00075560 \\ https://hal.inria.fr/inria-00075560}

Submitted on 24 May 2006

HAL is a multi-disciplinary open access archive for the deposit and dissemination of scientific research documents, whether they are published or not. The documents may come from teaching and research institutions in France or abroad, or from public or private research centers.
L'archive ouverte pluridisciplinaire HAL, est destinée au dépôt et à la diffusion de documents scientifiques de niveau recherche, publiés ou non, émanant des établissements d'enseignement et de recherche français ou étrangers, des laboratoires publics ou privés. 


\title{
RESOLUTION PAR UNE METHODE D'ELEMENTS FINIS DES EQUATIONS D'UN FLUIDE INCOMPRESSIBLE NON VISQUEUX EN GEOMETRIE AXISYM- METRIQUE. APPLICATION AU CALCUL D'UNE POMPE HELICE.
}

\author{
Jacques-Hervé SAIAC \\ CNAM - INRIA
}

Résumé

Nous présentons dans cette étude une méthode d'élément finis pour le calcul de l'écoulement d'un fluide incompressible, non visqueux, en géométrie axisymétrique. Notre approche utilise essentiellement une formulation fonction de courant, vitesse angulaire et tourbillon des équations d'Euler et nous avons résolu les problèmes stationnaires et instationnaires. Dans le cadre d'une application au calcul d'une pompe-hélice sur l'arrière corps d'un navire, nous avons développé un modèle de calcul complet de l'interaction entre le propulseur carenné et le corps du navire. Ce modèle utilise une version simple et rapide de la méthode des caractéristiques dans un contexte éléments finis. La solution stationnaire.de l'écoulement s'obtient par itérations de Picard. Les tests numériques ont mis en évidence la rapidité et la robustesse de la méthode. Des expériences réalisées au Bassin des Carènes ont révélé un très bon accord entre calcul et mesures.

\section{FINITE ELEMENT SOLUTIONS OF AXISYMMETRIC EULER EQUATIONS FOR AN INCOMPRESSIBLE AND INVISCID FLUID}

\section{ABSTRACT}

In this paper, we present a finite element method for the numerical solution of axisymmetric flows. The governing equations of the flow are the axisymmetric Euler equations. We use a stream-function angular velocity and vorticity formulation of these equations, and we consider the non stationary and the stationary problems.

For industrial applications, we have developed a general model which computes the flow past an annular airfoil and a duct propeller. It is able to take into account jumps of angular velocity and vorticity in order to model the flow in the presence of a propeller. Moreover we compute the complete flow around the after body of a ship and the interaction between a ducted propeller and the stern. In the stationnary case, we developped a simple and efficient version of the Characteristics / Finite element method. Numerical tests have shown that this last method leads to a very fast solver of the Euler equations. The numerical results are in good agreement with experimental data. 


\section{INTRODUCTION}

The stream-function and vorticity formulation of the Euler equations governing an incompressible and non viscous flow has been successfully used in two dimensional problems. In a finite element context it has been associated either with classical leap-frog or Crank-Nicolson time-differencing schemes [ 1,2$]$ or with the method of characteristics $[3,4,5]$.

It is well known that in the axisymmetric case, there is also a stream-function formulation of Euler equations. It uses the $\theta$ components, in cylindrical coordinates, of the vector potential, the velocity and the vorticity. The choice of this formulation has, in our case, many advantages. Among them we can
mention the following.

First, the axisymmetric flow is completely described by three scalar functions.

Moreover the incompressibility condition is exactly satisfied.

At last, from a computational point of view, this formulation gives a simple model leading to fast solvers well adapted to our purpose: "trial and error " procedures in engineering design.

Our model involve three equations. One elliptic equation for the stream function and two transport equations for the angular velocity and the vorticity. A finite element method using non uniform meshes has been chosen in order to get a general spatial discretization giving a soft treatment of geometry. Then, the main difficulty of the numerical solution of Euler equations is to write a good solver of the transport equations. Since for applications in "pump-jet" design, we have to model the convection of jumps of

angular velocity and vorticity, we need a robust method, especially well suited to difficult problems with
rough conditions. This has been the key-point of this work.

and [9] some finite element applications. The numerical methods in turbomachinery flows, and in [8] triangular meshes, direct solutions by Choleski factont work differs from the preceding by the choice of direct treatment of final choice of a stationary implementation ofition, elsewhere obtained through an iterative process. Our is the original part of this work.

This paper is organised as follows:

In sections 1 and 2 , we derive the mathematical formulations and the boundaries conditions of the problem. We precise the treatment of the Kutta-Joukovski condition.

In section 3 , we present the finice element spatial discretization and we give a convergence result in a simpler model case without "swirl".

The section 4 deals with time discretizations using Leap-Frog and Semi-Implicit Crank-Nicolson schemes. We derive theoretical stability results in both cases and we present some numerical tests showing the inability of this classical approach to model correctly the flow.

The following sections 5 and 6 are devoted to our implementation of the characteritics method giving the stationary solution of the flow by an iterative fixed-point algorithm.

At last. in section 7 . we present numerical results in the case of the complete model of a duct propeller. They reveal good agreement with experiments made by B. Goirand at the Bassin des Carenes
in Paris. 


\section{The mathematical model}

The general 3.dimensional Euler equations in cylindrical coordinates $r, \theta, z$ read :

$$
\begin{aligned}
\frac{\partial V_{r}}{\partial t}+V_{r} \frac{\partial V_{r}}{\partial r}+\frac{V_{\theta}}{r} \frac{\partial V_{r}}{\partial \theta}-\frac{V_{\theta}^{2}}{r}+V_{z} \frac{\partial V_{r}}{\partial z} & =-\frac{1}{\rho} \frac{\partial p}{\partial r} \\
\frac{\partial V_{\theta}}{\partial t}+V_{r} \frac{\partial V_{\theta}}{\partial r}+\frac{V_{\theta}}{r} \frac{\partial V_{\theta}}{\partial \theta}+\frac{V_{\theta} V_{r}}{r}+V_{z} \frac{\partial V_{\theta}}{\partial z} & =-\frac{1}{\rho r} \frac{\partial p}{\partial \theta} \\
\frac{\partial V_{z}}{\partial t}+V_{r} \frac{\partial V_{z}}{\partial r}+\frac{V_{\theta}}{r} \frac{\partial V_{z}}{\partial \theta}+V_{z} \frac{\partial V_{z}}{\partial z} & =-\frac{1}{\rho} \frac{\partial p}{\partial z}
\end{aligned}
$$

with

$$
\operatorname{div}(V)=\frac{1}{r} \frac{\partial}{\partial r}\left(r V_{r}\right)+\frac{1}{r} \frac{\partial V_{\theta}}{\partial \theta}+\frac{\partial V_{z}}{\partial z}=0
$$

$V_{r}, V_{\theta}, V_{z}$ are the components of the velocity, $\rho$ is the density of the fluid, $p$ is the pressure.

They reduce in the axisymmetric case to the following system

$$
\begin{aligned}
& \frac{\partial V_{r}}{\partial t}+V_{r} \frac{\partial V_{r}}{\partial r}+V_{z} \frac{\partial V_{r}}{\partial z}=\frac{V_{\theta}^{2}}{r}-\frac{1}{\rho} \frac{\partial p}{\partial r} \\
& \frac{\partial V_{\theta}}{\partial t}+V_{r} \frac{\partial V_{\theta}}{\partial r}+V_{z} \frac{\partial V_{\theta}}{\partial z}=-\frac{V_{\theta} V_{r}}{r} \\
& \frac{\partial V_{z}}{\partial t}+V_{r} \frac{\partial V_{z}}{\partial r}+V_{z} \frac{\partial V_{z}}{\partial z}=-\frac{1}{\rho} \frac{\partial p}{\partial z}
\end{aligned}
$$

with

$$
\frac{1}{r} \frac{\partial}{\partial r}\left(r V_{r}\right)+\frac{\partial V_{2}}{\partial z}=0
$$

Remark 1 :

We did not restrict ourselves to the case $V_{\theta}=0$. We just take the derivatives

$$
\frac{\partial}{\partial \theta}=0
$$

We introduce a stream-function $\psi_{\theta}$ such that the meridian velocity

$$
V_{M}=\left(V_{z}, V_{r}\right)
$$


can be written :

$$
V_{z}=\frac{1}{r} \frac{\partial\left(r \psi_{\theta}\right)}{\partial r}, V_{r}=-\frac{1}{r} \frac{\partial\left(r \psi_{\theta}\right)}{\partial z}
$$

Thus the zero-divergence condition $(2, d)$ is automatically satisfied.

Now, we consider the $\theta$-component of the vorticity vector

$$
\omega_{\theta}=\frac{\partial V_{r}}{\partial z}-\frac{\partial V_{z}}{\partial r}
$$

Equations $(2, a, b, c)$ lead through straight forward calculations to the following system in $\psi_{\theta}, V_{\theta}, \omega_{\theta}$.

$$
\begin{aligned}
&-\frac{\partial}{\partial z}\left(\frac{1}{r} \frac{\partial\left(r \psi_{\theta}\right)}{\partial z}\right)-\frac{\partial}{\partial r}\left(\frac{1}{r} \frac{\partial\left(r \psi_{\theta}\right)}{\partial r}\right)=\omega_{\theta} \\
& \frac{\partial\left(r V_{\theta}\right)}{\partial t}+V_{r} \frac{\partial\left(r V_{\theta}\right)}{\partial r}+V_{z} \frac{\partial\left(r V_{\theta}\right)}{\partial z}=0 \\
& \frac{\partial\left(\frac{\omega_{\theta}}{r}\right)}{\partial t}+V_{r} \frac{\partial\left(\frac{\omega_{\theta}}{r}\right)}{\partial r}+V_{2} \frac{\partial\left(\frac{\omega_{\theta}}{r}\right)}{\partial z}=\frac{1}{r^{2}} \frac{\partial\left(V_{\theta}^{2}\right)}{\partial z}
\end{aligned}
$$
work.

With the identities (3), the above system defines completely the flow. It is the basic model of this

\section{Remark 2 :}

Equation $(5, a)$ is a simple elliptic equation in $r \psi_{\theta}$.

Equation $(5, b)$ and $(5, c)$ appear as transport equations of $r V_{\theta}$ and $\frac{\omega_{\theta}}{r}$, respectively, along the stream lines, with the presence of a left hand term in $(5, c)$.

\section{The boundary conditions}

Let $\Omega$ denote in the sequel a bounded open set of $R^{2}$ with boundary $\Gamma$, such that for every point of coordinates $(z, r)$ in $\Omega$ we have :

$$
0<r_{0} \leq r \leq r_{1}
$$

The classical inviscid boundary condition

$$
u \cdot n=0
$$

leads to the following condition on $\psi_{\theta}$

$$
\operatorname{curl}\left(r \psi_{\theta}\right) \cdot n=0
$$


Thus we get

$$
\left.r \psi_{\theta}\right|_{\Gamma i}=c_{i}
$$

where the $c_{i}$ are constant, for each component $\Gamma_{i}$ of the boundary $\Gamma$.

\subsection{Model 1}

As a theoretical model, we consider the case of a simply connected domain $\Omega$ with the boundary condition

$$
\left.r \psi_{\theta}\right|_{\Gamma}=0
$$

\subsection{Model 2}

Now we turn to a more realistic case. $\Omega$ will denote the meridian section of an annular duct

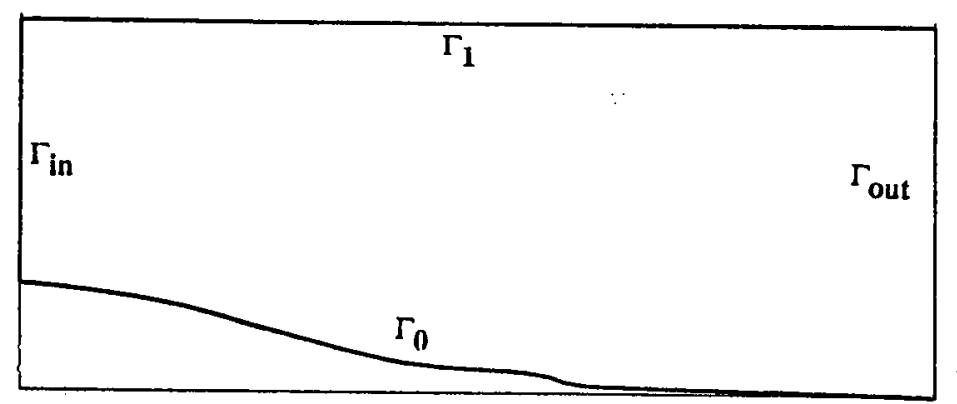

Figure 1.The axisymmetric duct

- $\Gamma_{0}$ and $\Gamma_{1}$ are supposed to be slipping walls.

- On the upstream boundary $\Gamma_{\text {in }}$, the velocity field is given. zero.

- On the downstream boundary $\Gamma_{\text {out }}$, we only suppose that the radial component of the velocity is

This model represents the flow around an axisymmetric body. The boundary $\Gamma_{1}$ is supposed far enough from $\Gamma_{0}$ to be an horizontal stream-surface. That leads to the following boundary conditions for $\psi_{\theta}, V_{\theta}$ and $\omega_{\theta}$ : 


\section{On $\Gamma_{\text {in }}, \Gamma_{0}$ and $\Gamma_{1}$.}

We deduce the values of $\psi_{\theta}, V_{\theta}$ and $\omega_{\theta}$ from the given velocity field. $r \psi_{\theta}$ being defined up to a constant, we are able to choose $r \psi_{\theta}=0$ on $\Gamma_{0}$. Then the law of $r \psi_{\theta}$ on $\Gamma_{i n}$ is completely known and we get the constant value $\mathrm{c}$ of $r \psi_{\theta}$ on $\Gamma_{1}$.

On $\Gamma_{\text {out }}$.

The condition $V_{r}=0$ leads to the homogenous Neumann boundary condition

$$
\left.\frac{\partial\left(r \psi_{\theta}\right)}{\partial n}\right|_{\Gamma_{\text {owt }}}=0
$$

\subsection{Model 3}

Let us now consider the same annular duct but with an axisymmetric airfoil shape body inside

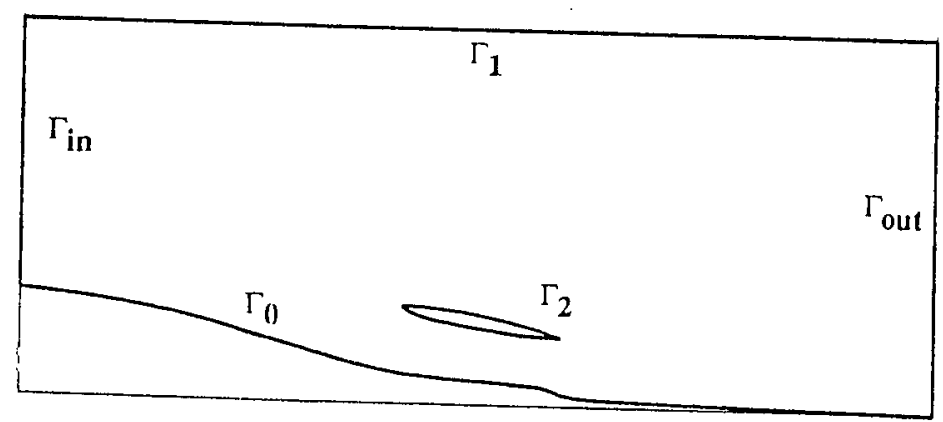

Figure 2.The complete model geometry

On the airfoil boundary $\Gamma_{2}$ we have the inviscid boundary condition.

$$
u \cdot n=0
$$

which leads to

$$
r \psi_{\left.\theta\right|_{\Gamma_{2}}}=c_{2}
$$

The problem is then to determine the physically correct value of the constant $c_{2}$. This has been done by using a Kutta Joukovski condition. This condition implies the equality of the static pressures at, the upper and the lower sides of the trailing edge. 

We made the computation in the following manner [ $F$. Hecht : private communication] : we looked
for a stream function $r \psi_{\theta}$ given by

$$
r \psi_{\theta}=\psi_{0}+\alpha \psi_{1}
$$
with $\psi_{0}$ solution of equation $(5$, a) at each time step, with the real boundary condition, except on
$\Gamma_{2}$ where we take

$$
\psi_{0 \mid r_{2}}=0
$$

and $\psi_{1}$, solution of the simple homogeneous equation

$$
-\frac{\partial}{\partial z}\left(\frac{1}{r} \frac{\partial \psi_{1}}{\partial z}\right)-\frac{\partial}{\partial r}\left(\frac{1}{r} \frac{\partial \psi_{1}}{\partial r}\right)=0
$$

with all the Dirichlet boundary conditions equal to zero except on $\Gamma_{2}$ where we take

$$
\psi_{\left.1\right|_{2}}=1
$$

The parameter $\alpha$ is then computed at each time step in order to satisfy the equality of the static pressures at the upper and lower sides of the trailing edge

$$
P_{s}^{+}=P_{s}^{-}
$$

i.e.

$$
\left|\operatorname{curl}\left(\psi_{0}+\alpha \psi_{1}\right)\right|^{2}+\left|\operatorname{curl}\left(\psi_{0}+\alpha \psi_{1}\right)\right|^{2}=\frac{2}{\rho}\left(P^{+}-P^{-}\right)
$$

where $P^{+}$and $P^{-}$are the pressures on the upper and lower sides of the trailing edge.

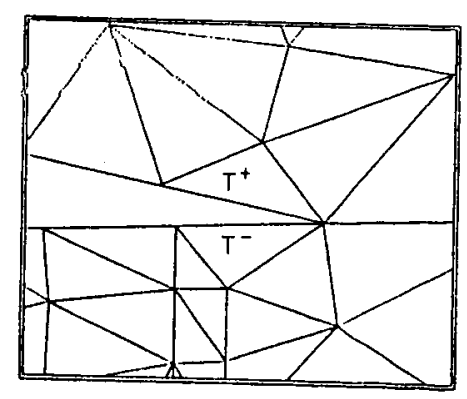

Figure 3.The numerical treatment of the Kutta - Joukovski condition. 
This quadratic equation in $\alpha$ has two solutions. The right one is the root for which the normal velocities are opposite.

\section{Finite element approximation}

In order to derive a finite element approximation of the problem, we need to introduce a variational form of the axisymmetric Euler equations.

\subsection{Basic concepts and function spaces}

Let $(.,:)$ denote the axisymmetric scalar product in $L^{2}(\Omega)$.

$$
(u, v)=\iint_{\Omega} u \cdot v \cdot r \cdot d r d z
$$

and

$$
\|\|_{0, \Omega} \text { the associated norm in } L^{2}(\Omega)
$$

For $m \in N$ and $p \in R$ with $1 \leq p \leq+\infty$ we define the Sobolev spaces

$$
W^{m, p}(\Omega)=\left\{v \in L^{p}(\Omega) ; \partial^{\alpha} v \in L^{p}(\Omega), \forall|\alpha| \leq m\right\}
$$

which is a Banach space for the norm

$$
\|v\|_{m, p, \Omega}=\left(\sum_{|\alpha| \leq m} \iint_{\Omega}\left|\partial^{\alpha} v(x)\right|^{p} r d r d z\right)^{\frac{1}{p}} \quad p<+\infty
$$

or

$$
\|v\|_{m, \infty, \Omega}=\sup _{|\alpha| \leq m}\left(\operatorname{supess}_{x \in \Omega}\left|\partial^{a} v(x)\right|\right) \quad p=+\infty
$$

We also provide $W^{m, p}(\Omega)$ with the following seminorm

$$
\begin{gathered}
|v|_{m, p, \Omega}=\left(\sum_{|\alpha|=m} \int_{\Omega}\left|\partial^{\alpha} v(x)\right|^{p} r d r d z\right)^{\frac{1}{p}} \quad \text { for } \quad p<+\infty \\
|v|_{m, \infty, \Omega}=\sup _{|\alpha| \leq m}\left(\operatorname{supess}_{x \in \Omega}\left|\partial^{\alpha} v(x)\right|\right) \quad \text { for } \quad p=+\infty
\end{gathered}
$$
In the special case $p=2$, we obtain the Hilbert spaces $H^{m}(\Omega)$ with the norm $\|\cdot\|_{m, \Omega}$ and the
senuinorm $|\cdot|_{m, \Omega}$. 
Let us introduce the bilinear form on $\left(H^{1}(\Omega)\right)^{2}$

$$
\begin{gathered}
a:(u, v) \rightarrow a(u, v) \\
a(u, v)=\iint_{\Omega} \frac{1}{r}\left(\frac{\partial u}{\partial r} \frac{\partial v}{\partial r}+\frac{\partial u}{\partial z} \frac{\partial v}{\partial z}\right) d r d z
\end{gathered}
$$

and the trilinear form on $W^{1, \infty}(\Omega) \times H^{1}(\Omega) \times L^{2}(\Omega)$

$$
\begin{gathered}
b:(u, v, w) \rightarrow(u, v, w) \\
b(u, v, w)=\iint_{\Omega}\left(\frac{\partial u}{\partial z} \frac{\partial v}{\partial r}-\frac{\partial u}{\partial r} \frac{\partial v}{\partial z}\right) \cdot w \cdot d r d z
\end{gathered}
$$

\subsection{Properties of the linear forms $a$ and $b$.}

\subsubsection{The linear form a}

Since for every point $(z, r)$ in $\Omega$ we supposed that :

$$
0<r_{0} \leq r \leq r_{1}
$$

the bilinear form a is, as in the two dimensional case, continuous in $\left(H^{1}(\Omega)\right)^{2}$ and $H_{0}^{1}(\Omega)-$ Elliptic Moreover we have the following inequalities

$$
\begin{gathered}
\frac{\alpha}{r_{1}^{2}}\|u\|_{1, r}^{2} \leq \frac{1}{r_{1}^{2}}|u|_{1, \Omega}^{2} \leq a(u, u) \quad \forall u \in H_{0}^{1}(\Omega) \\
|a(u, v)| \leq \frac{1}{r_{0}^{2}}|u|_{1, \Omega}|v|_{1, \Omega} \quad \forall u, v \in H^{1}(\Omega)
\end{gathered}
$$

\subsubsection{The form $b$}

The trilinear form $b$ satisfies the following properties

1) b is continuous in $W^{1, \infty}(\Omega) \times H^{1}(\Omega) \times L^{2}(\Omega)$

2) $\forall \mathrm{u} \in W_{0}^{1, \infty}(\Omega), \mathrm{v} \in H^{1}(\Omega)$ we have

$$
b(u, v, v)=b(u, v, u)=0
$$


Proof

$$
\text { 1) }|b(u, v, w)|=\left|\iint_{\Omega}\left(\frac{\partial u}{\partial z} \frac{\partial v}{\partial r}-\frac{\partial u}{\partial r} \frac{\partial v}{\partial z}\right) \cdot \frac{w}{r} \cdot r d r d z\right|
$$

so that :

$$
|b(u, v, w)| \leq \frac{1}{r_{0}}\left\|\frac{\partial u}{\partial z} \frac{\partial v}{\partial r}-\frac{\partial u}{\partial r} \frac{\partial v}{\partial z}\right\|_{0, \Omega}\|w\|_{0, \Omega}
$$

and

$$
|b(u, v, w)| \leq \frac{1}{r_{0}}|u|_{1, \infty, \Omega}|v|_{1, \Omega}|| w \|_{0, \Omega}
$$

2) The form $b$ is exactly the same as the form of the convective term in the two dimensional Euler equations and we refer to SAIAC [1] for the proof.

\subsection{Variational formulation}

Using a classical Green's formula, we obtain, in the case of model 1 , the following variational formulation of equations $(5 ; \mathrm{a}, \mathrm{b}, \mathrm{c})$

Find a function $\mathrm{t} \in[0, \mathrm{~T}] \rightarrow\left(\psi_{\theta}(t), v_{\theta}(t), \omega_{\theta}(t)\right) \in H_{0}^{1}(\Omega) \times H^{1}(\Omega) \times H^{1}(\Omega)$ such that :

$$
\begin{aligned}
a\left(r \psi_{\theta}(t), u\right) & =\left(\frac{\omega_{\theta}}{r}(t), u\right) \quad \forall u \in H_{0}^{1}(\Omega) \\
E \quad\left(\frac{d}{d t} r v_{\theta}(t), v\right) & =b\left(r \psi_{\theta}(t), r v_{\theta}(t), v\right) \quad \forall v \in H^{1}(\Omega) \\
\left(\frac{d}{d t} \frac{\omega_{\theta}}{r}(t), w\right) & =b\left(r \psi_{\theta}(t), \frac{\omega_{\theta}}{r}(t), w\right)+\left(\frac{1}{r^{2}} \frac{\partial}{\partial z}\left(v_{\theta}^{2}(t)\right), w\right) \quad \forall w \in H^{1}(\Omega)
\end{aligned}
$$

\subsection{Conservations properties of problem $\mathrm{E}$}

Using the fondamental property (29) of the trilinear form b we derive the following results :

1)

$$
\frac{1}{2} \frac{d}{d t}\left\|r v_{\theta}(t)\right\|_{0, \Omega}^{2}=b\left(r \psi_{\theta}(t), r v_{\theta}(t), r v_{\theta}(t)\right)=0 \quad \forall t \in[0, T]
$$

Thus we get the conservation of the $L^{2}$ norm of $r v_{\theta}(t)$

$$
\left\|r v_{\theta}(t)\right\|_{0, \Omega}=\left\|r v_{\theta}(0)\right\|_{0, \omega} \quad \forall t \in[0, T]
$$

2) $\quad \frac{1}{2} \frac{d}{d t}\left\|\frac{\omega_{\theta}}{r}(t)\right\|_{0, \Omega}^{2}=b\left(r \psi_{\theta}(t), \frac{\omega_{\theta}}{r}(t), \frac{\omega_{\theta}}{r}(t)\right)+\left(\frac{1}{r^{2}} \frac{\partial}{\partial z}\left(v_{\theta}^{2}(t)\right), \frac{\omega_{\theta}}{r}(t)\right)$ 
so that :

$$
\frac{d}{d t}\left\|\frac{\omega_{\theta}}{r}(t)\right\|_{0, \Omega} \leq \frac{1}{r_{0}^{2}}\left\|\frac{\partial}{\partial z}\left(v_{\theta}^{2}(t)\right)\right\|_{0, \Omega}
$$

and finally

$$
\left\|\frac{\omega_{\theta}}{r}(t)\right\|_{0, \Omega} \leq\left\|\frac{\omega_{\theta}}{r}(0)\right\|_{0, \Omega}+\frac{1}{r_{0}^{2}} \int_{0}^{t}\left\|\frac{\partial}{\partial z}\left(v_{\theta}^{2}(t)\right)\right\|_{0, \Omega} d t
$$

Remark : In the particular case of an initial value of $v_{\theta}$ equal to zero, $v_{\theta}$ remains null for all $t \in$ $[0, T]$ and the system $E$ reduces to two coupled equations involving $r \psi_{\theta}$ and $\frac{\omega_{\theta}}{r}$

Moreover in that case, we get

$$
\left\|\frac{\omega_{\theta}}{r}(t)\right\|_{0, \Omega}=\left\|\frac{\omega_{\theta}}{r}(0)\right\|_{0, \Omega} \quad \forall t \in[0, T]
$$

\subsection{Generalization}

3.5.1 In the case of model 2 the test function space in the first elliptic equation $(31, a)$ is replaced by the space $\mathrm{V}$ defined by :

$$
V=\left\{v \in H^{1}(\Omega) ; v=0 \text { on } \Gamma_{\text {in }} \cup \Gamma_{0} \cup \Gamma_{1}\right\}
$$

The unknown stream-function $r \psi_{\theta}$ satisfies the following boundary conditions

$r \psi_{\theta}$ given on $\Gamma_{i n}$

$r \psi_{\theta}=0$ on $\Gamma_{0}$ and $r \psi_{\theta}=c$ (given constant) on $\Gamma_{1}$

$\frac{\partial\left(r \psi_{\theta}\right)}{\partial n}=0$ on $\Gamma_{\text {out }}$

3.5.2 In the case of model 3, we moreover have to take into account a Kutta Joukovski condition (see paragraph 2.3). We made the computations as follows :

The stream function $\psi_{1}$ has been computed once and for all at the beginning of the program.

It does not depend on time. Then we just have to solve one elliptic equation at each time step to get the complete stream-function. 


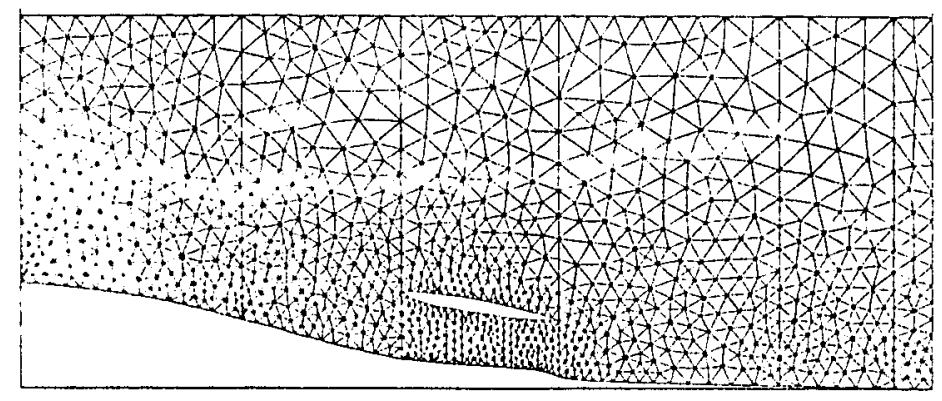

Figure 4. The computational mesh in the case of the complete model.

1,$1 ; . .11:$ the two finite dimensional spaces such that $U_{h} \subset W_{0}^{1, \infty}(\Omega)$ and $V_{h} \subset W^{1, \infty}(\Omega)$.

Wr apprnximatc the continuous problem $(E)$ by the following approximate problem $\left(E_{h}\right)$.

lint a function $\mathrm{t} \in[0, \mathrm{~T}] \rightarrow\left(\psi_{\theta, h}(t), v_{\theta, h}(t), \omega_{\theta, h}(t)\right) \in U_{h} \times V_{h} \times V_{h}$ satisfying for all $\mathrm{t} \in[0, \mathrm{~T}]$

$$
\begin{aligned}
\therefore\left(r \psi_{, h}(t), u_{h}\right) & =\left(\frac{\omega_{\theta, h}}{r}(t), u_{h}\right) \quad \forall u_{h} \in U_{h} \\
& \left(\frac{d}{d t} r v_{\theta, h}(t), v_{h}\right)=b\left(r \psi_{\theta, h}(t), r v_{\theta, h}(t), v_{h}\right) \quad \forall v_{h} \in V_{h} \\
\left(\frac{d}{d t} \frac{\omega_{i, h}}{r}(t), w_{h}\right) & =b\left(r \psi_{\theta, h}(t), \frac{\omega_{\theta, h}}{r}(t), w_{h}\right)+\left(\frac{1}{r^{2}} \frac{\partial}{\partial z}\left(v_{\theta, h}^{2}(t)\right), w_{h}\right) \quad \forall w_{h} \in V_{h}
\end{aligned}
$$

\subsubsection{Cinservation properties of problem $E_{h}$}

W. Li, for the approximate solutions $r \psi_{\theta, h}, r v_{\theta, h}$ and $\frac{w_{\theta, h}}{r}$ the same bounds as for the exact ‥lution

Fir inntinew:

$$
\left\|r v_{\theta, h}(t)\right\|_{0, \Omega}=\left\|r v_{\theta, h}(0)\right\|_{0, \Omega} \quad \forall t \in[0, T]
$$




\subsubsection{A first convergence result}

Let us consider the simplest model problem $E^{*}$ in the particular case of $V_{\theta}=0$, (flow without "swirl"). and $\omega_{\theta}$.

The corresponding approximate problem $E_{h}^{*}$ reduces to the following system of two equations in $\psi_{\theta}^{\prime}$

$E_{h}^{*}$

$$
a\left(r \psi_{\theta, h}(t), u_{h}\right)=\left(\frac{\omega_{\theta, h}}{r}(t), u_{h}\right) \quad \forall u_{h} \in U_{h}
$$

$$
\left(\frac{d}{d t} \frac{\omega_{\theta, h}}{r}(t), v_{h}\right)=b\left(r \psi_{\theta, h}(t), \frac{\omega_{\theta, h}}{r}(t), v_{h}\right) \quad \forall v_{h} \in V_{h}
$$

Assume that $\left(\psi_{\theta}, \omega_{\theta}\right)$ solution of the problem $E^{*}$ belongs to the space

$$
L^{\infty}\left(0, T ;\left[W^{k+1, \infty}(\Omega) \cup W_{0}^{1, \infty}(\Omega)\right] \times W^{k+1, \infty}(\Omega)\right)
$$

Then under classical hypotheses of finite element interpolation we get the following error bound

$$
\left|\psi_{\theta}^{\prime}(t)-\psi_{\theta, h}(t)\right|_{1, \Omega}+\left\|\omega_{\theta}(t)-\omega_{\theta, h}(t)\right\|_{0, \Omega} \leq C h^{k}
$$

Proof :

The proof follows the same lines as the proof of the convergence of the finite element method in the two dimensional case, see SAIAC [ 1 ]. In fact the form b here is exactly the same as in the two dimensional case and there are just slight differences in the expressions of the bilinear form a and the scalar product.

These differences are very easy to handle since we can assume that hypotheses (6) hold.

\section{Remark : The general case.}

The general case is more tricky because of the term

$$
\left(\frac{1}{r^{2}} \frac{\partial}{\partial z}\left(v_{\theta, h}^{2}(t)\right), w_{h}\right)
$$

in the equation $(38, c)$.

We did not succeed already to proove the convergence of the finite element scheme in that case. 


\section{Time discretization}

Let us choose a positive integer $\mathbf{N}$, let $\Delta t$ denote the corresponding time-step

$$
\Delta t=\frac{T}{N}
$$

and $\left(t_{n}\right)$ the subdivision of $[0, T]$

$$
t_{n}=n \cdot \Delta t \quad \text { for } \quad 0 \leq n \leq N
$$

let $\psi_{\theta, h}^{n}, v_{\theta, h}^{n}$ and $\omega_{\theta, h}^{n}$ denote approximations of $\psi_{\theta}\left(t_{n}\right), v_{\theta}\left(t_{n}\right)$ and $\omega_{\theta}\left(t_{n}\right)$ respectively.

\subsection{The Leap-frog scheme}

The leap-frog scheme for the problem $\mathbf{E}$ can be written as follows

$$
\begin{aligned}
a\left(r \psi_{\theta, h}^{n}, u_{h}\right) & =\left(\frac{\omega_{\theta, h}^{n}}{r}, u_{h}\right) \quad \forall u_{h} \in U_{h} \\
\left(r v_{\theta, h}^{n+1}-r v_{\theta, h}^{n-1}, v_{h}\right) & =2 \Delta t \quad b\left(r \psi_{\theta, h}^{n}, r v_{\theta, h}^{n}, v_{h}\right) \quad \forall v_{h} \in V_{h} \\
\left(\frac{\omega_{\theta, h}^{n+1}}{r}-\frac{\omega_{\theta, h}^{n-1}}{r}, w_{h}\right) & =2 \Delta t \quad\left[b\left(r \psi_{\theta, h}^{n}, \frac{\omega_{\theta, h}^{n}}{r}, w_{h}\right)+\left(\frac{1}{r^{2}} \frac{\partial}{\partial z}\left(v_{\theta, h}^{n}\right)^{2}, w_{h}\right)\right] \quad \forall w_{h} \in V_{h}
\end{aligned}
$$

with $\psi_{\theta, h}^{0}, v_{\theta, h}^{0}$ and $\omega_{\theta, h}^{0}$ given and $\psi_{\theta, h}^{1}, v_{\theta, h}^{1}$ and $\omega_{\theta, h}^{1}$ solutions of

$$
\begin{aligned}
a\left(r \psi_{\theta, h}^{1}, u_{h}\right) & =\left(\frac{\omega_{\theta, h}^{1}}{r}, u_{h}\right) \quad \forall u_{h} \in U_{h} \\
\left(r v_{\theta, h}^{1}-r v_{\theta, h}^{0}, v_{h}\right) & =\Delta t \quad b\left(r \psi_{\theta, h}^{0}, r v_{\theta, h}^{0}, v_{h}\right) \quad \forall v_{h} \in V_{h} \\
\left(\frac{\omega_{\theta, h}^{1}}{r}-\frac{\omega_{\theta, h}^{0}}{r}, w_{h}\right) & =\Delta t \quad\left[b\left(r l_{\theta, h}^{0}, \frac{\omega_{\theta, h}^{0}}{r}, w_{h}\right)+\left(\frac{1}{r^{2}} \frac{\partial}{\partial z}\left(v_{\theta, h}^{0}\right)^{2}, w_{h}\right)\right] \quad \forall u_{h}^{\prime} \in V_{h}
\end{aligned}
$$

The stability of the Leap-Frog scheme follows from the next lemma.

\subsubsection{Lemma}

Under the following stability hypotheses :

$$
\text { 1) } \quad c \Delta t\left[\frac{1}{h}\left|r \psi_{\theta, h}^{n}\right|_{1, \infty, \Omega}+\frac{1}{r^{4}}\left|r v_{\theta, h}^{n}\right|_{1, \infty, \Omega}\right]<1 \quad \forall n \in 0, N
$$


2) there exists a constant $A>0$ such that

$$
\frac{C}{h}\left|r \psi_{\theta, h}^{n}-r \psi_{\theta, h}^{n-1}\right|_{1, \infty, \Omega}+\frac{c}{r^{4}}\left(\left|r v_{\theta, h}^{n}\right|_{1, \infty, \Omega}+\left|r v_{\theta, h}^{n+1}\right|_{1, \infty, \Omega}\right)<A .
$$

We have the following bound for every $n=0, \ldots \mathrm{N}$

$$
\left\|r v_{\theta, h}^{n}\right\|_{0, \Omega}^{2}+\left\|\frac{\omega_{\theta, h}^{n}}{r}\right\|_{0, \Omega}^{2} \leq C\left(\left\|r v_{\theta, h}^{0}\right\|_{0, \Omega}^{2}+\left\|r v_{\theta, h}^{1}\right\|_{0, \Omega}^{2}+\left\|\frac{\omega_{\theta, h}^{0}}{r}\right\|_{0, \Omega}^{2}+\left\|\frac{\omega_{\theta, h}^{1}}{r}\right\|_{0, \Omega}^{2}\right)
$$

\section{Proof :}

Let us introduce

$$
\begin{gathered}
S_{n}=\left\|r v_{\theta, h}^{n}\right\|_{0, \Omega}^{2}+\left\|r v_{\theta, h}^{n+1}\right\|_{0, \Omega}^{2}+\left\|\frac{w_{\theta, h}^{n}}{r}\right\|_{0, \Omega}^{2}+\left\|\frac{w_{\theta, h}^{n+1}}{r}\right\|_{0, \Omega}^{2} \\
-2 \Delta t . b\left(r \psi_{\theta, h}^{n}, r v_{\theta, h}^{n}, r v_{\theta, h}^{n+1}\right)-2 \Delta t . b\left(r \psi_{\theta, h}^{n}, \frac{\omega_{\theta, h}^{n}}{r}, \frac{\omega_{\theta, h}^{n+1}}{r}\right)-2 \Delta t\left(\frac{1}{r^{2}} \frac{\partial}{\partial z}\left(v_{\theta, h}^{n}\right)^{2}, \frac{\omega_{\theta, h}^{n+1}}{r}\right)
\end{gathered}
$$

We follow, as in ref [] the energy method used by Richtmyer and Morton.

We have :

$$
\left|b\left(r \psi_{\theta, h}^{n}, r v_{\theta, h}^{n}, r v_{\theta, h}^{n+1}\right)\right| \leq C\left|r \psi_{\theta, h}^{n}\right|_{1, \infty, \Omega}\left|r v_{\theta, h}^{n}\right|_{1, \Omega}|| r v_{\theta, h}^{n+1} \|_{0, \Omega}
$$

and by use of the inverse inequality :

$$
\left|b\left(r \psi_{\theta, h}^{n}, r v_{\theta, h}^{n}, r v_{\theta, h}^{n+1}\right)\right| \leq \frac{C}{h}\left|r \psi_{\theta, h}^{n}\right|_{1, \infty, \Omega}\left\|r v_{\theta, h}^{n}\right\|_{0, \Omega}|| r v_{\theta, h}^{n+1} \|_{0, \Omega}
$$

Similarly, we get :

$$
\left|b\left(r \psi_{\theta, h}^{n}, \frac{\omega_{\theta, h}^{n}}{r}, \frac{\omega_{\theta, h}^{n+1}}{r}\right)\right| \leq \frac{C}{h}\left|r \psi_{\theta, h}^{n}\right|_{1, \infty, \Omega}\left\|\frac{\omega_{\theta, h}^{n}}{r}\right\|_{0, \Omega}\left\|\frac{\omega_{\theta, h}^{n+1}}{r}\right\|_{0, \Omega}
$$

and

$$
\left|\left(\frac{1}{r^{2}} \frac{\partial}{\partial z}\left(v_{\theta, h}^{n}\right)^{2}, \frac{\omega_{\theta, h}^{n+1}}{r}\right)\right| \leq \frac{c}{r_{0}^{4}}\left|r v_{\theta, h}^{n}\right|_{1, \infty, \Omega}\left\|r v_{\theta, h}^{n}\right\|_{0, \Omega}\left\|\frac{\omega_{\theta, h}^{n+1}}{r}\right\|_{0, \Omega}
$$


so that

$\left(1-C \frac{\Delta t}{h}\left|r \psi_{\theta, h}^{n}\right|_{1, \infty, \Omega}-c \frac{\Delta t}{r^{4}}\left|r v_{\theta, h}^{n}\right|_{1, \infty, \Omega}\right) \cdot\left(\left\|r v_{\theta, h}^{n}\right\|_{0, \Omega}^{2}+\left\|r v_{\theta, h}^{n+1}\right\|_{0, \Omega}^{2}+\left\|\frac{\omega_{\theta, h}^{n}}{r}\right\|_{0, \Omega}^{2}+\left\|\frac{\omega_{\theta, h}^{n+1}}{r}\right\|_{0, \Omega}^{2}\right)$

$\leq S_{n} \leq\left(1+C \frac{\Delta t}{h}\left|r \psi_{\theta, h}^{n}\right|_{1, \infty, \Omega}+c \frac{\Delta t}{r^{4}}\left|r v_{\theta, h}^{n}\right|_{1, \infty, \Omega}\right)\left(\left\|r v_{\theta, h}^{n}\right\|_{0, \Omega}^{2}+\left\|r v_{\theta, h}^{n+1}\right\|_{0, \Omega}^{2}+\left\|\frac{\omega_{\theta, h}^{n}}{r}\right\|_{0, \Omega}^{2}+\left\|\frac{\omega_{\theta, h}^{n+1}}{r}\right\| \|_{0, \Omega}^{2}\right)$

But we also have

$$
\begin{gathered}
S_{n}-S_{n-1}=\left\|r v_{\theta, h}^{n+1}\right\|_{0, \Omega}^{2}-\left\|r v_{\theta, h}^{n-1}\right\|_{0, \Omega}^{2}+\left\|\frac{\omega_{\theta, h}^{n+1}}{r}\right\|_{0, \Omega}^{2}-\left\|\frac{\omega_{\theta, h}^{n-1}}{r}\right\|_{0, \Omega}^{2} \\
-2 \Delta t . b\left(r \psi_{\theta, h}^{n}, r v_{\theta, h}^{n}, r v_{\theta, h}^{n+1}\right)+2 \Delta t b\left(r \psi_{\theta, h}^{n-1}, r v_{\theta, h}^{n-1}, r v_{\theta, h}^{n}\right) \\
-2 \Delta t . b\left(r \psi_{\theta, h}^{n}, \frac{\omega_{\theta, h}^{n}}{r}, \frac{\omega_{\theta, h}^{n+1}}{r}\right)+2 \Delta t . b\left(r \psi_{\theta, h}^{n-1}, \frac{\omega_{\theta, h}^{n-1}}{r}, \frac{\omega_{\theta, h}^{n}}{r}\right) \\
-2 \Delta t \cdot\left(\frac{1}{r^{2}} \frac{\partial}{\partial z}\left(v_{\theta, h}^{n}\right)^{2}, \frac{\omega_{\theta, h}^{n+1}}{r}\right)+2 \Delta t \cdot\left(\frac{1}{r^{2}} \frac{\partial}{\partial z}\left(v_{\theta, h}^{n-1}\right)^{2}, \frac{\omega_{\theta, h}^{n}}{r}\right)
\end{gathered}
$$

using then :

$$
\left\|r v_{\theta, h}^{n+1}\right\|_{0, \Omega}^{2}-\left\|r v_{\theta, h}^{n-1}\right\|_{0, \Omega}^{2}=2 \Delta t . b\left(r \psi_{\theta, h}^{n}, r v_{\theta, h}^{n}, r v_{\theta, h}^{n-1}+r v_{\theta, h}^{n+1}\right)
$$

and

$$
\left\|\frac{\omega_{\theta, h}^{n+3}}{r}\right\|_{0, \Omega}^{2}-\left\|\frac{\omega_{\theta, h}^{n-1}}{r}\right\|_{0, \Omega}^{2}=2 \Delta t \cdot\left[b\left(r \psi_{\theta, h}^{n}, \frac{\omega_{\theta, h}^{n}}{r}, \frac{\omega_{\theta, h}^{n-1}+\omega_{\theta, h}^{n+1}}{r}\right)+\left(\frac{1}{r} \frac{\partial}{\partial z}\left(v_{\theta, h}^{n}\right)^{2}, \frac{\omega_{\theta, h}^{n-1}}{r}+\frac{\omega_{\theta, h}^{n+1}}{r}\right)\right]
$$

we get

$$
\begin{aligned}
& S_{n}-S_{n-1}=2 \Delta t {\left[b\left(r, l_{\theta, h}^{n}-r \psi_{\theta, h}^{n-1}, r v_{\theta, h}^{n}, r v_{\theta, h}^{n-1}\right)+b\left(r \psi_{\theta, h}^{n}-r \psi_{\theta, h}^{n-1}, \frac{\omega_{\theta, h}^{n}}{r}, \frac{\omega_{\theta, h}^{n-1}}{r}\right)\right.} \\
&\left.+\left(\frac{1}{r^{2}} \frac{\partial}{\partial z}\left(v_{\theta, h}^{n}\right)^{2}, \frac{\omega_{\theta, h}^{n-1}}{r}\right)+\left(\frac{1}{r^{2}} \frac{\partial}{\partial z}\left(v_{\theta, h}^{n-1}\right)^{2}, \frac{\omega_{\theta, h}^{n}}{r}\right)\right]
\end{aligned}
$$


So that we get

$$
\begin{gathered}
\left.S_{n}-S_{n-1} \leq \frac{2 c \Delta t}{h}\left|r \psi_{\theta, h}^{n}-r \psi_{\theta, h}^{n-1}\right|_{1, \infty, \Omega} \quad\left[\left\|r v_{\theta, h}^{n}\right\|_{0, \Omega}\left\|r v_{\theta, h}^{n-1}\right\|_{0, \Omega}+\left\|\frac{\omega_{\theta, h}^{n}}{r}\right\|_{0, \Omega}\right]\left\|\frac{\omega_{\theta, h}^{n-1}}{r}\right\|_{0, \Omega}\right] \\
+\frac{2 c \Delta t}{r_{0}^{4}}\left[\left.\left|r v_{\theta, h}^{n}\right|_{1, \infty, \Omega}\left\|r v_{\theta, h}^{n}\right\|_{0, \Omega}\left\|\frac{\omega_{\theta, h}^{n-1}}{r}\right\|\right|_{0, \Omega}+\left|r v_{\theta, h}^{n-1}\right|_{1, \infty, \Omega}\left\|r v_{\theta, h}^{n-1}\right\|\left\|_{0, \Omega}\right\| \frac{\omega_{\theta, h}^{n}}{r} \|_{0, \Omega}\right] \\
S_{n}-S_{n-1} \leq\left[\frac{c \Delta t}{h}\left|r \psi_{\theta, h}^{n}-r \psi_{\theta, h}^{n-1}\right|_{1, \infty, \Omega}+\frac{c \Delta t}{r^{4}}\left(\left|r v_{\theta, h}^{n}\right|_{1, \infty, \Omega}+\left|r v_{\theta, h}^{n-1}\right|_{1, \infty, \Omega}\right)\right] . \\
{\left[\left\|r v_{\theta, h}^{n}\right\|_{0, \Omega}^{2}+\left\|r v_{\theta, h}^{n-1}\right\|_{0, \Omega}^{2}+\left\|\frac{\omega_{\theta, h}^{n}}{r}\right\|_{0, \Omega}^{2}+\left\|\frac{\omega_{\theta, h}^{n-1}}{r}\right\|_{0, \Omega}^{2}\right]}
\end{gathered}
$$

and by use of the stability hypotheses we derive

$$
\begin{gathered}
\left\|r v_{\theta, h}^{n}\right\|_{0, \Omega}^{2}+\left\|r v_{\theta, h}^{n-1}\right\|_{0, \Omega}^{2}+\left\|\frac{\omega_{\theta, h}^{n}}{r}\right\|_{0, \Omega}^{2}+\left\|\frac{\omega_{\theta, h}^{n-1}}{r}\right\|_{0, \Omega}^{2} \leq K S_{0} \\
+A K \Delta t \sum_{m=1}^{n}\left[\left\|r v_{\theta, h}^{m}\right\|_{0, \Omega}^{2}+\left\|r v_{\theta, h}^{m-1}\right\|_{0, \Omega}^{2}+\left\|\frac{\omega_{\theta, h}^{m}}{r}\right\|_{0, \Omega}^{2}+\left\|\frac{\omega_{\theta, h}^{m-1}}{r}\right\|_{0, \Omega}^{2}\right]
\end{gathered}
$$

and the result

\subsection{A Semi implicit scheme of order two}

This scheme is a semi-implicit CRANK - NICOLSON scheme. It can be written as follow in a finite element context :

$$
\begin{aligned}
a\left(r \psi_{\theta, h}^{n}, u_{h}\right) & =\left(\frac{\omega_{\theta, h}^{n}}{r}, u_{h}\right) \quad \forall u_{h} \in U_{h} \\
a\left(r \dot{\psi}_{\theta, h}^{\frac{n+1}{2}}, u_{h}\right) & =\left(\frac{\omega_{\theta, h}^{n}}{r}, u_{h}\right)+\frac{\Delta t}{2}\left[b\left(r \psi_{\theta, h}^{n}, \frac{\omega_{\theta, h}^{n}}{r}, u_{h}\right)+\left(\frac{1}{r^{2}} \frac{\partial}{\partial z}\left(v_{\theta, h}^{n}\right)^{2}, u_{h}\right)\right] \quad \forall u_{h} \in U_{h} \\
\left(r v_{\theta, h}^{n+1}-r v_{\theta, h}^{n}, v_{h}\right) & =\frac{\Delta t}{2} b\left(r \psi_{\theta, h}^{\frac{n+1}{2}}, r v_{\theta, h}^{n}+r v_{\theta, h}^{n+1}, v_{h}\right) \quad \forall v_{h} \in V_{h} \\
\left(\frac{\omega_{\theta, h}^{n+1}}{r}-\frac{\omega_{\theta, h}^{n}}{r}, w_{h}\right) & =\frac{\Delta t}{2}\left[b\left(r \psi_{\theta, h}^{\frac{n+1}{2}}, \frac{\omega_{\theta, h}^{n}}{r}+\frac{\omega_{\theta, h}^{n+1}}{r}, w_{h}\right)+\left(\frac{1}{r^{2}}\left(\frac{\partial}{\partial z}\left(v_{\theta, h}^{n}\right)^{2}+\frac{\partial}{\partial z}\left(v_{\theta, h}^{n+1}\right)^{2}\right), w_{h}\right)\right] \forall w_{h} \in V_{h}(45,
\end{aligned}
$$

This scheme is of order two in time and it satisfies the following stability property

Let us assume that it exists a constant $\mathrm{A}$ such that we get the inequality : 


$$
\left\|\frac{1}{r^{2}} \frac{\partial}{\partial z}\left(v_{\theta, h}^{n}\right)^{2}+\frac{1}{r^{2}} \frac{\partial}{\partial z}\left(v_{\theta, h}^{n+1}\right)^{2}\right\|_{0, \Omega} \leq A
$$

the preceding scheme is stable and we easily get :

$$
\begin{gathered}
\left\|r v_{\theta, h}^{n}\right\|_{0, \Omega}=\left\|r v_{\theta, h}^{0}\right\|_{0, \Omega} \quad \forall n=0,1, \ldots N \\
\left\|\frac{\omega_{\theta, h}^{n}}{r}\right\|_{0, \Omega} \leq\left\|\frac{\omega_{\theta, h}^{0}}{r}\right\|_{0, \Omega}+\frac{A T}{2}
\end{gathered}
$$

\subsection{Methods of characteristics}

For the application of the method of characteristics to the transport equations $(5, \mathrm{~b})$ and $(5, \mathrm{c})$ in a finite element context we refer to El Dabaghi and Sajac [6]

\subsection{Numerical tests}

The numerical tests have shown that the explicit Leap-frog scheme requires very small time steps in order to satisfy the stability condition. The semi-implicit scheme is better. Although it is more costly for each time step, it can work with much larger $\Delta t$ and globally, it is faster.

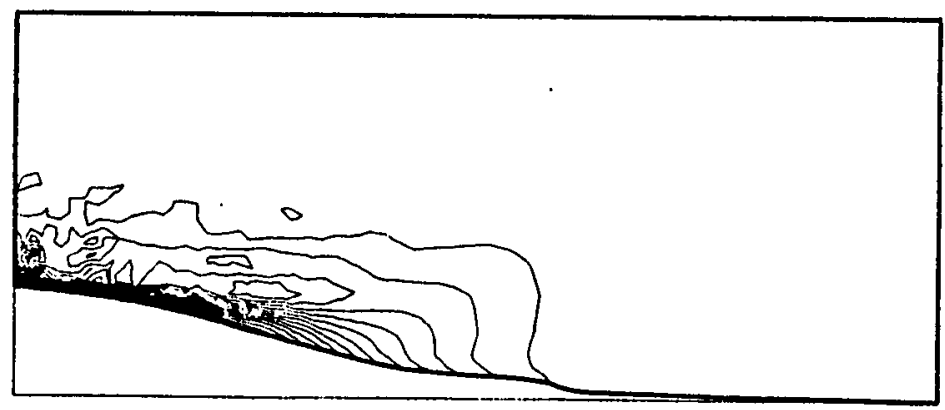

Figure 5.The vorticity $\left(\frac{\omega_{\theta}}{r}\right)$ field at $t=1$ computed by the Leap-frog scheme with $\Delta t=0.001$

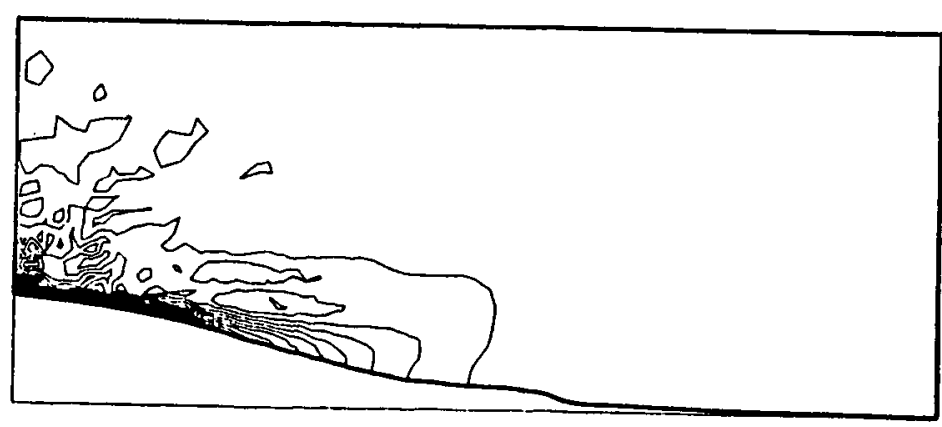

Figure 6.The vorticity $\left(\frac{\omega_{\theta}}{r}\right)$ field at $t=1$ computed by the semi-implicit scheme with $\Delta t=0.01$ 
However the best results for the time dependent transport equation were obtained by the use of the characteristics method.See a comparison of this schemes in ref [6]

\section{An iterative method for the stationary solution}

In order to get the stationary solution of EULER equations (5.a, b, c) we can of course use the time discretization schemes quoted above. But it is a better choice with respect to numerical stability and computational time to use the following simple version of the characteristics method. This implementation essentially uses our easy knowledge of the stream lines, which is the case for plane or axisymmetric flow problems.

Let us consider the simplest model without swirl (i.e. with $V_{\theta}=0$ ) to explain the method. In that case the problem reduces to the problem $\mathrm{E}^{*}$, the vortex $\frac{\omega_{\theta}}{r}$ is simply convected along the stream lines $r \varphi_{\theta}^{\prime}=$ cste.

Thus to detemine $\frac{\omega_{\theta}}{r}$ at any point $\mathrm{x}$ of the domain $\Omega$ we just have to find on which stream lines lies the point $x$. Then we go back along this stream-line to the entry point on the upstream boundary where the value of the vortex is given.

We can summarize the computational process by the following iteration method.

Suppose that $\psi_{\theta, h}^{0}$ is given at the time $t_{0}$. Then for any $\mathrm{n} \geq 0$, define $\psi_{\theta, h}^{n+1}$ from $\psi_{\theta, h}^{n}$ by :

$$
a\left(r \psi_{\theta, h}^{n+1}, u_{h}\right)=\left(\frac{\omega_{\theta, h}^{n}}{r}, u_{h}\right)=\left(\omega_{i}\left(\psi_{\theta, h}^{n}\right), u_{h}\right) \quad \forall u_{h} \in U_{h}
$$

where $\omega_{i}$ is the numerical function, defined from the given upstream boundary values of the flow, which gives the funciional law between the values of $r \psi_{\theta}$ and those of $\frac{\omega_{\theta}}{r}$.

More generally, we can consider the family of algorthms

$\psi_{\theta, h}^{0}$ given at $t_{0}$

Then $\psi_{\theta, h}^{n+1}$ is computed from $\psi_{\theta, h}^{n}$ by :

$$
a\left(r \psi_{\theta, h}^{n+1}, u_{h}\right)=a\left(r \psi_{\theta, h}^{n}, u_{h}\right)-\rho\left(a\left(r \psi_{\theta, h}^{n}, u_{h}\right)-\left(\omega_{i}\left(r \psi_{\theta, h}^{n}\right), u_{h}\right)\right) \quad \forall u_{h} \in U_{h}
$$

If $\rho=1$ we recover $(49)$

Moreover, if $\omega_{i}$ is differentiable, we should be able to solve the problem by a Newton's method such as the following :

$\psi_{\theta, h}^{0}$ given at $t_{0}$

$$
a\left(r \psi_{\theta, h}^{n+1}, u_{h}\right)-\left(\omega_{i}^{\prime}\left(r \psi_{\theta, h}^{n}\right) \cdot r \psi_{\theta, h}^{n+1}, u_{h}\right)=\left(\omega_{i}\left(r \psi_{\theta, h}^{n}\right), u_{h}\right)-\left(\omega_{i}^{\prime}\left(r \psi_{\theta, h}^{n}\right) \cdot r \psi_{\theta, h}^{n}, u_{h}\right) \quad \forall u_{h} \in U_{h}
$$


This kind of iterations has been studied by many authors. One's can find an interesting discussion in Glowinski [10]. See also Eydeland and Turkington [11]

First of all we have the following theoretical result. See Brezis, Sibony [12 ]

Let us consider the following non linear problem.

Find $u \in U$ such that

$$
a(u, v)=(\omega(u), v) \quad \forall v \in U
$$

Where $\mathbf{a}$ is a bilinear, continuous and strongly elliptic form which satisfies :

$$
\begin{aligned}
& a\|u\|^{2} \leq a(u, u) \quad \forall u \in U \text { with } \quad \alpha>0 \\
& |a(u, v)| \leq M\|u\| v \| \quad \forall u \text { and } v \in U
\end{aligned}
$$

and $\omega$ is a non linear operator in $\mathrm{U}$

Let us define $A: U \rightarrow U$ by

$$
(A(u), v)=a(u, v)-(\omega(u), v) \quad \forall u \quad \text { and } \quad v \in U
$$

We have the following result

\section{Theorem :}

Suppose $A$ is Lipschitz continuous on the bounded sets of $U$ and suppose that $A$ is strongly elliptic, i.e there exists a constant $k>0$ such that

$$
\left(A\left(u_{2}\right)-A\left(u_{1}\right), u_{2}-u_{1}\right) \geq k\left\|u_{2}-u_{1}\right\|^{2} \quad \forall u_{1} \quad \text { and } \quad u_{2} \in U
$$

Then the problem ( 52 ) has a unique solution. Moreover the following iteration

$$
\begin{aligned}
& u^{0} \text { given } \\
& u^{n+1} \text { defined from } u^{n} \text { by }
\end{aligned}
$$

$$
a\left(u^{n+1}, v\right)=a\left(u^{n}, v\right)-\rho\left(a\left(u^{n}, v\right)-\left(w\left(u^{n}\right), v\right)\right) \quad \forall v \in U
$$


converge to the solution u of ( 52$)$ for every constant $\rho$ satisfying

$$
0<\rho<\rho_{M}
$$

$\rho_{M}$ being a positive constant depending on $u^{0}$ in general

Let us make some comments on the ellipticity condition (56).

In our case, it implies there exists a positive number $\mathrm{k}$ such that, for all $u_{1}$ and $u_{2}$ in $U$ we have :

$$
a\left(u_{2}-u_{1}, u_{2}-u_{1}\right)-\left(\omega_{i}\left(u_{2}\right)-\omega_{i}\left(u_{1}\right), u_{2}-u_{1}\right) \geq k\left\|u_{2}-u_{1}\right\|^{2}
$$

But a is strongly elliptic, with :

$$
a\left(u_{2}-u_{1}, u_{2}-u_{1}\right) \geq \alpha\left\|u_{2}-u_{1}\right\|^{2}
$$

Then the inequality will be obtained if we can ensure that

$$
\left(\omega_{i}\left(u_{2}\right)-\omega_{i}\left(u_{1}\right), u_{2}-u_{1}\right) \leq 0 \quad \forall u_{1} \quad \text { and } \quad u_{2} \in U
$$

or if we can suppose that $\omega_{i}$ satisfies a Lipschitz condition

$$
\left\|\omega_{i}\left(u_{2}\right)-\omega_{i}\left(u_{1}\right)\right\| \leq L\left\|u_{2}-u_{1}\right\|
$$

with a Lipschitz constant $\mathrm{L}$ such that $\mathrm{L}<\alpha$

Let us remark that in this last case, it is easy to proove the convergence of the iterations (49) by a contraction argument .

We don't know whether the condition (59) is a necessary condition for the existence or the stability of the flow. And we refer to A rnold [13] for further considerations on stability of stationary solutions of Euler equations. Let us however point out the following problem :

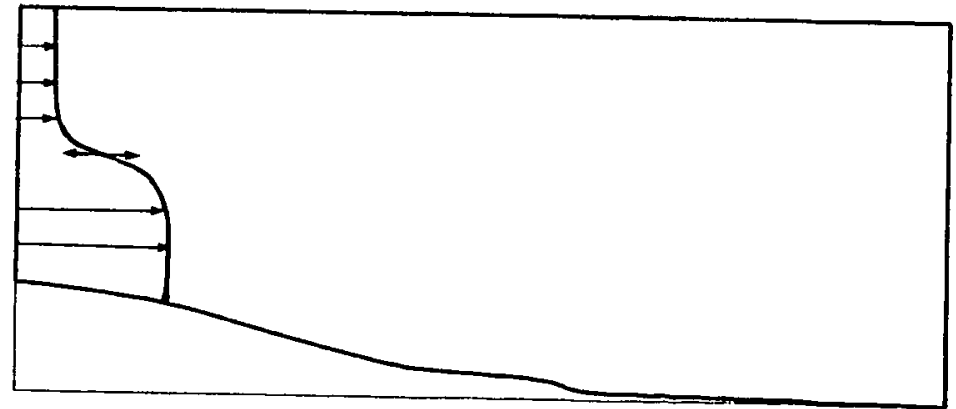

Figure 7. 
In the case of a flow with a given velocity profile such that $\omega_{i}^{\prime}$ is not bounded, we do have instability of the flow. And this is one of the main difficulty of the modelization of a propeller .

\section{Fixed - point iteration algorithm versus time - dependent approach .}

Let us denote by $\psi_{\theta, h}$ the stationary solution of the approximate problem $\mathrm{E}_{h}$. When the fixed-point algorithm is convergent, we get the following error bound for every iteration $n=0, \ldots N$

with $\mathrm{k} \leq 1$

$$
\left\|\psi_{\theta, h}^{n}-\psi_{\theta, h}\right\|_{1, \Omega} \leq C k^{n}\left\|\psi_{\theta, h}^{0}-\psi_{\theta, h}\right\|
$$

The convergence rate depends on the value of $k$, but we shall get for sufficiently large $N$

$$
\left\|\psi_{\theta, h}^{n}-\psi_{\theta, h}\right\|_{1, \Omega} \leq \epsilon
$$

whatever $\epsilon$ be. Then the global error is only a finite element interpolation error.

On the contrary, in a time dependent approach, each iteration corresponds to a time step. We solve a differential equation of the following kind .

$$
\frac{d}{d t} \psi_{\theta, h}=T\left(\psi_{\theta, h}\right)
$$

The best error bound we can get, by the use of the Gronwall Lemma, is the following :

$$
\left\|\psi_{\theta, h}^{n}-\psi_{\theta, h}\left(t_{n}\right)\right\|_{1, \Omega} \leq C \exp \left(A t_{n}\right) \quad\left[\left\|\psi_{\theta, h}^{0}-\psi_{\theta, h}(0)\right\|+h^{k}\right]
$$

Let us then suppose that the stationary solution is obtained at the time $T=t_{n}$ we then have the following inequality :

$$
\left\|\psi_{\theta, h}^{N}-\psi_{\theta, h}\right\|_{1, \Omega} \leq \operatorname{Cexp}(A T) \quad\left[\left\|\psi_{\theta, h}^{0}-\psi_{\theta, h}^{\prime}(0)\right\|+h^{k}\right]
$$

The initial error and the interpolation error are multiplied by a factor exp ( AT) which is quichly growing with $\mathbf{T}$. This is an heuristic reason why the fixed point iteration algorithm gives much better results that a time dependent approach when one's is only interested by the stationary solution

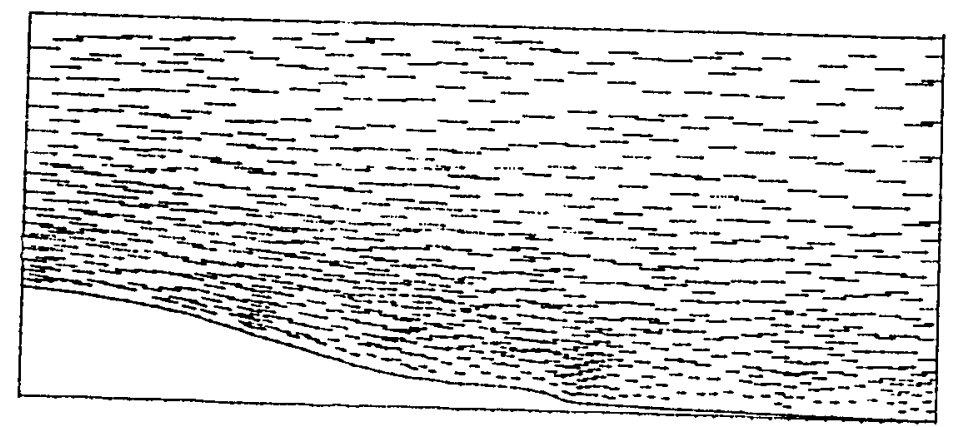

Figure 8.The velocity field of the stationary solution computed by our iterative method. 


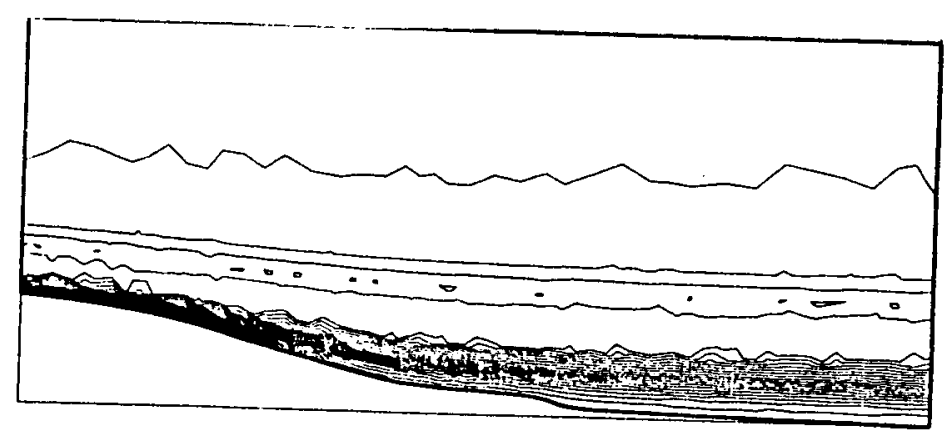

Figure 9.The vorticity $\left(\frac{\omega_{\theta}}{r}\right)$ field of the stationary solution computed by our iterative method.

\section{Modelization of a Duct Propeller .}

In order to modelize the presence of stators and rotors, we introduce jumps of the angular velocity $V_{\theta}$ and, for the rotors only, a jump of the pressure .

Let us say a few words about the computation of the pressure in our model. Pressures are convected along the stream lines from the upstream boundary to the downstream boundary. Then, when a stream line go accross a rotor, we add a jump of pressure in order to modelize the propeller effect. That increases the velocity in the duct through the Kutta-Joukovski condition. And we also need, in that case, to introduce some viscosity effects at the trailing edge of the duct.

After many numerical experiments and computational works, we determined two practical solutions:

First, we introduce some amount of vorticity at the trailing edge in order to maintain the jump of axial velocity up and down to the trailing edge of the duct. The physically correct value for that jump was choosen as follow:

$$
\left[\omega_{\theta}\right]=\frac{1}{\rho V_{z}} \frac{\partial P}{\partial r}
$$

This solution gives good results but it may lead to computational instabilities .

The second solution prevents instabilities. We make a first computation to determine the geometry of the stream line passing by the trailing edge point. Then we completely compute the flow with this imposed stream line .

Both methods have been compared and give similar results.

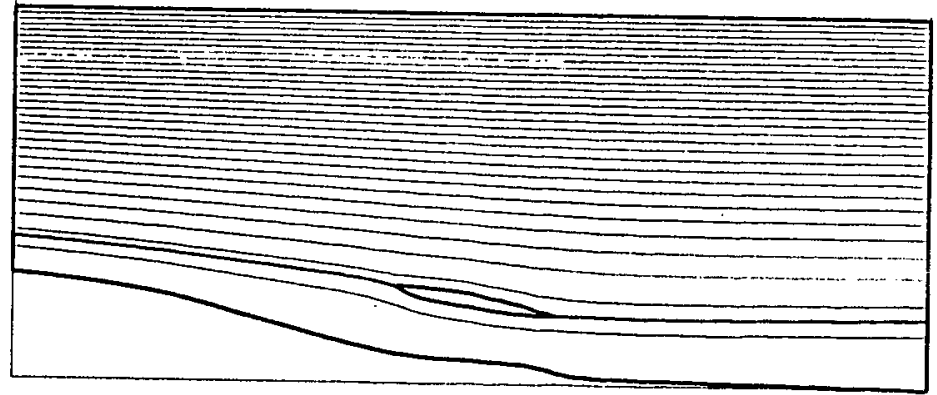

Figure 10.The stream lines of the stationary solution computed by our iterative method in the case of a duct propeller. 


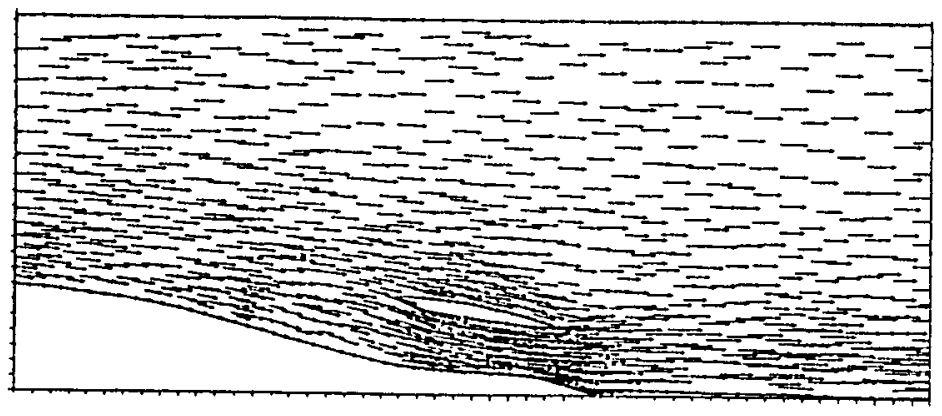

Figure 11.The velocity field of the stationary solution computed by our iterative method in the case of a duct propeller.

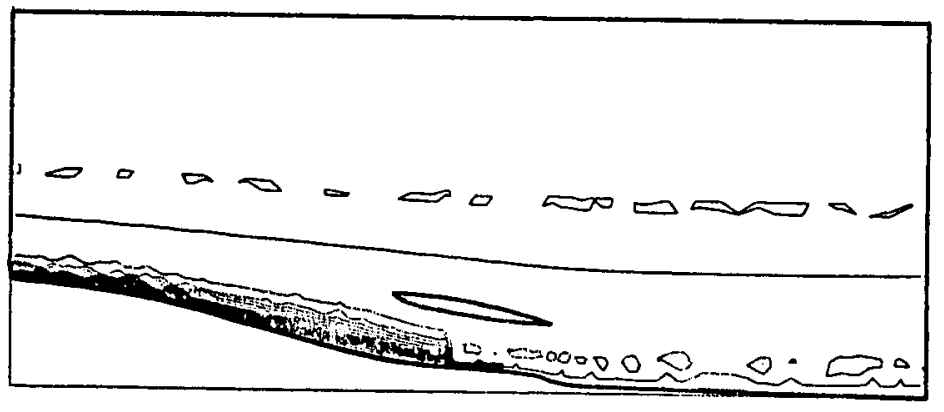

Figure 12.The vorticity $\left(\frac{\omega_{\theta}}{r}\right)$ field of the stationary solution computed by our iterative method in the case of a duct propeller. 

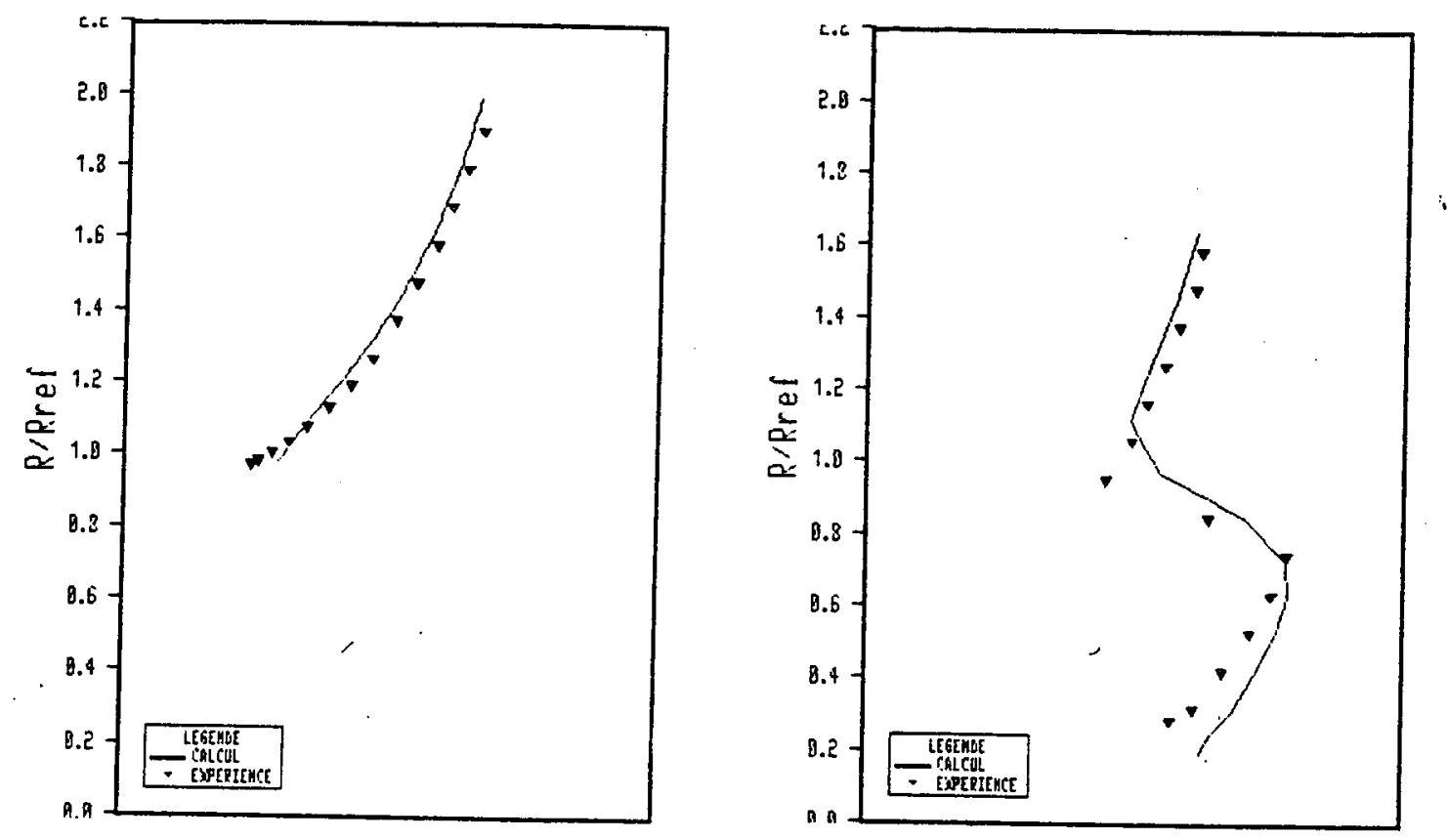

Figure 13. Comparison between computed and experimental velocity profiles before and after the propeller made by B. Goirand at the Bessin des Carenes in Paris.

More detailled discussions, complete tests and a comparison with real experiments made at the Bassin des Carenes in PARIS by B. Goirand are to appear .

\section{Conclusion}

In this paper, we presented a stable, precise, and very fast solver, based on the characteristics method, of the stationary axisymmetric Euler equation. Its stability and computing time perfomances are well adapted to "trial and error" procedures in engineering design. This scheme has been successfully used to compute an internal-external axisymmetric flow and to determine the whole propulsive performances, specially duct thrust and interaction betwen propulsor and stern. Comparisons with experiments made at the Bassin des Carenes in Paris have shown very good agreement between measures and calculus.

We are now developing a finite element blade to blade flows calculation in order to produce an automatic complete quasi $3 \mathrm{~d}$ solver. Our purpose is again to obtain a low time consuming, simple, stable, numerical code in order to use it in a engineering design context. 
References

1. Saiac, J.H. : Finite Element Method for time - dependent Euler Equation, Math. Method in the Appl. Sci., 5, 22-39, 1983.

2. Saiac, J.H. : On numerical solutions of the time-dependent Euler equations for incompressible flow, Int. j. Numer. Methods fluids, vol 5, 637-656, 1985.

3. Pironneau, 0 .: On the transport diffusion algorithm and its application to the Navier-Stokes Equations, Num. Math., 8, 309-332, 1980.

4. Bardos, C., Bercovier, M. and Pironneau, O.: The Vortex Method with finite elements, Math.Comp., $36,153,1981$.

5. El Dabaghi, F. , Pironneau, O. : Stream Vectors in the Three Dimensional Aerodynamics, Num.Math.48,pp. 561-589, 1986.

6. El Dabaghi, F., Saiac,J .H . Characteristic's and time dependent methods for solving the 3.D incompressible EULER equations by a stream-vector vorticity formulation, to appear in Hydrosoft Computational Mechanics Publications in 1988.

7. Habashi, W.G. : Numerical Methods for Turbomachinery, in C.Taylor and K.Morgan (eds.), Recent Advances in Numerical Methods in Fluids, Pineridge Press, Swansea, Wales, 1980.

8. Habashi, W.G. and Hafez M.M. : Finite Element Solutions of Transonic External and Internal Flow Calculations, ASME Pap 83-GT-35, New York 83.

9. Hirsch, Ch. and Warzee, G. : A Finite Element Methods for Through Flow Calculations in Turbomachines, ASME J. Fluids Eng., 98 : 403-421, 1976.

10. Glowinski, R. : Numerical Methods for Nonlinear Variational Problems,199-206. Springer - Verlag.

11. Eydeland, A. and Turkington, B. : A Computational Method of Solving Free-Boundary Problems in Vortex Dynamics, J.C.P., 78, 194-214, 1988.

12. Brezis, H. and Sibony, M. : Methodes d'approximation et d'iteration pour les operateurs monotones. Arch. Ration. Mech. Anal. 28, 59-82 1968.

13. Arnol'd, V. : Mathematical Methods of Classical Mechanics. Spinger-Verlag, 1978.

Imprimé en France

par

l'Institut National de Recherche en Informatique et en Automatique 
. 